\title{
A randomized approach to space debris footprint characterization *
}

\author{
Alessandro Falsone* Fabio Noce* Maria Prandini * \\ * Dipartimento di Elettronica, Informazione e Bioingegneria, \\ Politecnico di Milano, Italy. \\ e-mail: alessandro.falsone@polimi.it,fabio.noce@mail.polimi.it, \\ prandini@elet.polimi.it
}

\begin{abstract}
This paper studies the problem of characterizing the $4 \mathrm{D}$ (space cross time) region of the airspace that will be occupied by a space debris during an uncontrolled reentry, with the final goal of supporting the air traffic controllers in re-routing the air traffic when such an event occurs. The problem is formulated in terms of a chance-constrained optimization program, which is solved via a simulation-based method. The approach is comparatively evaluated against the so-called covariance propagation method recently proposed in the literature, emphasizing how some of the limitations of the latter method are overcome.
\end{abstract}

\section{INTRODUCTION}

Since the beginning of the space era the number of satellites grew significantly and so did the number of them which ceased their operations and now are orbiting around Earth without the possibility to be controlled from ground stations. Together with upper stage rocket bodies, and fragments generated by collisions with meteoroids or other artificial satellites, they constitute the so-called space debris population.

When a debris orbit decays, mainly due to atmospheric friction, it can reenter the atmosphere and pose risk to public safety, striking people and properties on the ground. During a controlled reentry, the spatial debris is maneuvered so as to make it reenter the Earth atmosphere avoiding high density fly zones and strike the ground in the ocean or over an uninhabited area. During an uncontrolled reentry, instead, the debris cannot be maneuvered and, hence, may cause serious risk for the population on the ground as well as for the air traffic. Studies in the literature on uncontrolled reentries have been focusing almost exclusively on the possible impact in terms of casualties on the population on the ground, see e.g. Pardini and Anselmo [2013], Anselmo and Pardini [2013], Pardini and Anselmo [2005], Frank et al. [2005], Weaver et al. [2011]. As a matter of fact, despite of the high aircraft vulnerability (in the case of an aircraft flying at a speed of 700 kilometers per hour even an impact with a small debris, hypothetically at rest in the air, can cause a severe damage, Pardini and Anselmo [2013]), only a few contributions have been focusing on the impact on air traffic, see Patera [2008] and Ailor and Wilde [2008].

Up today, accurate and reliable models to describe uncontrolled space debris reentry are not available, due to the complexity of the phenomenon. When a satellite ceases its operation, it typically continues to orbit around the Earth until the atmospheric drag reduces its speed making it lower its semi-major axis. Typical speed of an orbiting

* Research supported by the European Commission under the MoVeS project, FP7-ICT-2009-257005. satellite is about $7 \mathrm{~km} / \mathrm{s}$ and, as it enters the Earth atmosphere, it encounters a steadily increasing aerodynamic load that causes a sequence of failures thus generating multiple fragments, Ailor and Wilde [2008]. Though this fragmentation is far from instantaneous, we refer to it as breakup instant, meaning the instant when the reentering body experiences a major breakup (i.e. the first massive breakup) . In Weaver et al. [2011], Frank et al. [2005] it is shown that all fragments evolve with a ballistic trajectory, only subject to gravity and aerodynamic forces.

Observations have shown that the major breakup event happens at an altitude of about $78 \mathrm{~km}$ Ailor and Wilde [2008] with an uncertainty range from $\pm 10 \mathrm{~km}$ to $\pm 20 \mathrm{~km}$, Weaver et al. [2011], Frank et al. [2005], Pardini and Anselmo [2005]. So, fragments generated from this first breakup are distributed over a range of altitudes, thus increasing the spread of debris footprint along the direction of motion. Following Ailor and Wilde [2008], which suggests that the spread of debris footprint caused by breakup altitude uncertainty can be reduced if the object is observed during the major breakup, we focus only on fragment dispersion after the breakup phase.

Besides the complex nature of the breakup process, even post-breakup trajectories of fragments are subject to strong uncertainties. In particular, the aerodynamic drag acting on a fragment depends on the ballistic coefficient, which is uncertain, and on the atmospheric density, that is affected by modeling errors (Saunders et al. [2009], Pardini and Anselmo [2013]). Local wind is pointed out as a source of uncertainty in FAA [2011], Ailor and Wilde [2008], due to its effect on the debris trajectories after the breakup process, when the debris enters the low atmosphere. The horizontal component of the velocity vector is in fact typically dominated by the wind that can cause a crosstrack dispersion of the debris, Ailor and Wilde [2008], the impact being higher on those fragments with a lower ballistic coefficient, FAA [2011].

All this motivated the adoption of a probabilistic approach 
in Weaver et al. [2011], Frank et al. [2005] and in this paper as well.

Our goal is to propose a novel method for determining the debris footprint, i.e., the minimum size 4D (space cross time) region of the airspace where all debris trajectories except a set of predefined probability $\varepsilon \in(0,1)$ can be confined. More specifically, we introduce a simulationbased approach where the problem of the $\varepsilon$-footprint computation is formulated as a chance-constrained optimization problem and then solved through a randomized method, which reduces to generating a certain number of realizations of the reentry trajectory and imposing that these realizations belong to the footprint. The obtained $\varepsilon$-footprint can be used by air traffic controllers to define no-fly zones and redefine the aircraft flight plans so as to guarantee that they do not enter such zones. Note that the larger is a no-fly zone the more difficult is finding a feasible solution to the re-routing problem: $\varepsilon$ can then play the role of a tuning parameter so as to compromise between the admissible risk level and the size of the no-fly zone: 0 risk but a large no-fly zone if $\varepsilon=0$, and maximum risk but a zero volume no-fly zone if $\varepsilon=1$.

The proposed simulation-based method is compared with the analytic covariance propagation method recently proposed in Reyhanoglu and Alvarado [2013].

The paper unfold as follows. In Section 2 we focus on a model for single-fragment trajectory prediction after the entrance in the Earth atmosphere. Based on this model, we characterize the $4 \mathrm{D}$ debris footprint and describe the proposed simulation-based method for its computation in Section 3. After briefly recalling the covariance propagation method in Section 4.1, we compare the two methods in Section 4.2. Finally, in Section 5, we draw some conclusions and suggest some interesting directions of future research.

\section{MODEL DESCRIPTION}

For the debris model we adopt the three-degrees of freedom model of a falling object over a rotating planet recently proposed in Reyhanoglu and Alvarado [2013].

Let $\boldsymbol{x}=[x, y, z]^{\prime}$ represent the position of the falling fragment with respect to a topocentric horizon coordinate system at the breakup instant. The frame, from now on called ENZ (East-North-Zenith), is centered at $\left(\vartheta_{0}, \varphi_{0}\right)$ on the Earth surface (initial longitude: $\vartheta_{0}$; initial latitude: $\left.\varphi_{0}\right)$, the $x y$ plane is the local horizon (i.e. the plane tangent to the Earth at the origin), the $x$-axis points eastward, the $y$-axis points northward and the $z$-axis is directed towards the zenith. In this way, fragment reentry after breakup has $\boldsymbol{x}_{0}=\left[0,0, z_{0}\right]^{\prime}$ as initial position, with $z_{0}$ representing the altitude of breakup.

With reference to the ENZ frame, the equations of motion describing the debris trajectory are given by:

$$
\dot{\boldsymbol{x}}=\boldsymbol{v}
$$

$$
\dot{\boldsymbol{v}}=-\boldsymbol{a}_{d}-g \boldsymbol{e}_{3}-2 \boldsymbol{\omega} \times \boldsymbol{v}-\boldsymbol{\omega} \times\left[\boldsymbol{\omega} \times\left(\boldsymbol{x}+R_{e} \boldsymbol{e}_{3}\right)\right]+\boldsymbol{\xi}
$$

where $\boldsymbol{v}=\left[v_{x}, v_{y}, v_{z}\right]^{\prime}$ is the velocity, $\boldsymbol{a}_{d}$ is the atmospheric drag deceleration, $\boldsymbol{\omega}=\left[0, \omega_{e} \cos \left(\varphi_{0}\right), \omega_{e} \sin \left(\varphi_{0}\right)\right]^{\prime}$ is the angular velocity vector of the ENZ frame (Earth rotation rate: $\left.\omega_{e}=7.2921 \times 10^{-5} \mathrm{rad} / \mathrm{s}\right)$ and $\boldsymbol{e}_{3}=[0,0,1]^{\prime}$.

Constant $R_{e}$ represents the Earth mean radius $\left(R_{e}=\right.$ $\left.6.3728 \times 10^{6} \mathrm{~m}\right)$, whereas according to the inverse square gravity model, the gravitational acceleration is given by

$$
g=g_{g}\left(\frac{R_{e}}{R_{e}+z}\right)^{2},
$$

where $g_{g}=9.81 \mathrm{~m} / \mathrm{s}^{2}$ is the gravitational acceleration on the ground. The atmospheric drag deceleration $\boldsymbol{a}_{d}$ acting on the fragment can be expressed as

$$
\boldsymbol{a}_{d}=\frac{1}{2} \frac{\rho(z)}{\beta} v_{r} \boldsymbol{v}_{r}
$$

where $\rho(z)$ is the atmosphere density as a function of the altitude, $\boldsymbol{v}_{r}=\boldsymbol{v}-\boldsymbol{w}$ is the debris speed relative to the wind velocity $\boldsymbol{w}$, and $v_{r}=\left\|\boldsymbol{v}_{r}\right\|$ is the magnitude of $\boldsymbol{v}_{r}$. Parameter $\beta$ represents the ballistic coefficient and is given by $\beta=\frac{m}{C_{d} A}$, where $m$ is the mass of the debris, $A$ its crosssectional area, and $C_{d}$ the aerodynamics coefficient.

Finally, $\boldsymbol{\xi}$ is a random acceleration vector that accounts for modeling errors and disturbances.

\section{PROBABILISTIC FOOTPRINT COMPUTATION: A SIMULATION-BASED METHOD}

In this section, we present a randomized approach to determine the footprint of a debris fragment, which rests on the multiple simulations of its reentry trajectory.

The debris dispersion at a certain time instant $t$ will be confined within an ellipsoidal set

$$
\mathcal{E}_{\varepsilon}(A, \boldsymbol{c})=\left\{\boldsymbol{x} \in \Re^{3}:(\boldsymbol{x}-\boldsymbol{c})^{\prime} A(\boldsymbol{x}-\boldsymbol{c}) \leq 1\right\},
$$

finitely parameterized through vector $c$ and matrix $A$ respectively representing the center of the ellipsoid and the positive definite symmetric matrix defining its shape. The problem of computing the ellipsoid $\mathcal{E}_{\varepsilon}(A, \boldsymbol{c})$ with minimum volume that contains all possible debris trajectories except for a set of probability at most $\varepsilon$ can be formalized in terms of the following chance-constrained optimization problem:

$$
\begin{aligned}
\min _{A, \boldsymbol{c}} & \sqrt{\operatorname{det} A^{-1}} \\
\text { subject to: } & A=A^{\prime} \succ 0 \\
& \mathcal{P}\left\{\delta \in \Delta: \boldsymbol{x}_{\delta}(t) \in \mathcal{E}_{\varepsilon}(A, \boldsymbol{c})\right\} \geq 1-\varepsilon
\end{aligned}
$$

where $\boldsymbol{x}_{\delta}(t)$ denotes the position of the debris at time $t$, when the uncertainty affecting the debris reentry trajectory is $\delta$. The uncertainty vector $\delta$ can account for various sources of uncertainties (parameters, initial condition, disturbances) as discussed in the introduction, and takes values in some uncertainty set $\Delta$ according to a probability distribution $\mathcal{P}$. Sensible choices for $\mathcal{P}$ are suggested in Weaver et al. [2011], Frank et al. [2005].

If we let $\left(A^{\star}, c^{\star}\right)$ be the solution to $(3)$, then

$$
\mathcal{E}_{\varepsilon}^{\star}=\left\{\boldsymbol{x} \in \Re^{3}:\left(\boldsymbol{x}-\boldsymbol{c}^{\star}\right)^{\prime} A^{\star}\left(\boldsymbol{x}-\boldsymbol{c}^{\star}\right) \leq 1\right\}
$$

is the minimum volume ellipsoid that represents the $3 \mathrm{D}$ footprint associated with the violation parameter $\varepsilon$.

Chance-constrained optimization problems like (3) are known to be difficult to solve, Prèkopa [1995, 2003], except for specific cases like when the involved probability distribution is Gaussian. We next show how to approximately solve problem (3) via a randomized method, called the scenario approach, Calafiore and Campi [2005, 2006], Campi and Garatti [2008], Campi et al. [2009], Campi and Garatti [2011], which reduces the chance-constrained optimization problem to an optimization problem with standard (i.e., 
non probabilistic) constraints, while providing guarantees on the chance-constrained feasibility of the obtained solution.

To this purpose we first reformulate problem (3) as

$$
\min _{A, c} \log \operatorname{det} A^{-1}
$$

subject to: $A=A^{\prime} \succ 0$

$$
\mathcal{P}\left\{\delta \in \Delta: \boldsymbol{x}_{\delta}(t) \in \mathcal{E}_{\varepsilon}(A, \boldsymbol{c})\right\} \geq 1-\varepsilon,
$$

where the cost $\log \operatorname{det} A^{-1}$ to be minimized is convex as a function of $A$, Boyd [2004]. Convexity of both the cost and the set appearing under the probability $\mathcal{P}$ in the chanceconstraint is in fact needed for providing theoretical guarantees on the quality of the scenario solution.

The main concept of the scenario approach is that solvability of the optimization problem (4) can be achieved by extracting a finite number $N$ of realizations of the uncertainty vector $\delta$ and replacing the constraint in probability with the $N$ constraints associated with the extracted $\delta$ 's uncertainty instances.

This leads to the following convex optimization problem with a finite number of constraints:

$$
\begin{aligned}
\min _{A, \boldsymbol{c}} & \log \operatorname{det} A^{-1} \\
\text { subject to: } & A=A^{\prime} \succ 0 \\
& \boldsymbol{x}_{\delta^{(i)}}(t) \in \mathcal{E}_{\varepsilon}(A, \boldsymbol{c}), i=1,2, \ldots, N,
\end{aligned}
$$

where $\delta^{(i)}, i=1, \ldots, N$ are extracted independently from $\Delta$ according to the distribution $\mathcal{P}$.

As opposed to (4), (5) is a convex optimization problem with a finite number of constraints and can be addressed by computing first the convex hull of all data points $\boldsymbol{x}_{t}\left(\delta^{(i)}\right)$, and then applying Khachiyan's algorithm to find the minimum volume ellipsoid containing the convex hull, Todd and Yildirim [2007], Sun and Freund [2004].

The fact that the scenario solution is feasible also for the original problem is guaranteed by the following theorem, whose proof is given in Campi and Garatti [2008].

Theorem 1. Select a "violation parameter" $\varepsilon \in(0,1)$ and a "confidence parameter" $\eta \in(0,1)$. If $N$ is such that

$$
\sum_{i=0}^{d}\left(\begin{array}{c}
N \\
i
\end{array}\right) \varepsilon^{i}(1-\varepsilon)^{N-i} \leq \eta
$$

where $d$ denotes the number of optimization variables in (5), then, with probability no smaller than $1-\eta$, the solution $\left(A_{N}^{\star}, \boldsymbol{c}_{N}^{\star}\right)$ to the scenario optimization problem (5) satisfies

$$
\mathcal{P}\left\{\delta \in \Delta: \boldsymbol{x}_{\delta}(t) \in \mathcal{E}_{\varepsilon}\left(A_{N}^{\star}, \boldsymbol{c}_{N}^{\star}\right)\right\} \geq 1-\varepsilon .
$$

Note that the result on the feasibility of the scenario solution holds with a certain confidence $1-\eta$. This is because the scenario solution depends on the extracted uncertainty instances and it may then happen that a bad multi-sample (e.g., all $\delta^{(i)}$ 's equal) is extracted and the feasibility property does not hold. However, this becomes more and more unlikely as $N$ increases and the probability $\eta$ of this unfortunate event can be set as small as $10^{-10}$ (i.e., zero in practice) without growing too much the sample size $N$. Indeed, the explicit bound for $N$ satisfying (6)

$$
N \geq \frac{d+1+\ln (1 / \eta)+\sqrt{2(d+1) \ln (1 / \eta)}}{\varepsilon}
$$

derived in Alamo et al. [2010] shows that the dependence on $\eta$ is logarithmic.

Though extremely powerful, this randomized approach may lead to conservative results, i.e., to a solution with an actual violation that is much smaller than $\varepsilon$. This is quite intuitive: it is possible that a few "outliers" causing a significant increment of the cost function are extracted among the $\delta$ 's uncertainty instances. To avoid this problem, it would be useful to have a simple procedure for discarding such extractions without affecting the guarantees provided by Theorem 1. In Campi and Garatti [2011], a variant of the scenario method that includes constraint removal is proposed and feasibility of the obtained solution is proven. The associated scenario program is as follows

$$
\min _{A, \boldsymbol{c}} \log \operatorname{det} A^{-1}
$$

subject to: $A=A^{\prime} \succ 0$

$$
\boldsymbol{x}_{\delta^{(i)}}(t) \in \mathcal{E}_{\varepsilon}(A, \boldsymbol{c}), i \in\{1,2, \ldots, N\} \backslash I_{r},
$$

where $I_{r}=\left\{i_{1}, \ldots, i_{k}\right\} \subset\{1,2, \ldots, N\}$ is the set of indices of the uncertainty instances that are removed so as to improve the cost (i.e. reduce the volume of the ellipsoid).

Theorem 2. Select a "violation parameter" $\varepsilon \in(0,1)$, a "confidence parameter" $\eta \in(0,1)$ and an "empirical violation parameter" $\alpha \in[0, \epsilon)$. If $N$ is such that

$$
\left(\begin{array}{c}
\lfloor\alpha N\rfloor+d \\
\lfloor\alpha\rfloor
\end{array}\right) \sum_{i=0}^{\lfloor\alpha N\rfloor+d}\left(\begin{array}{c}
N \\
i
\end{array}\right) \varepsilon^{i}(1-\varepsilon)^{N-i} \leq \eta,
$$

where $d$ denotes the number of optimization variables in (5), then, if we set $k=\lfloor\alpha N\rfloor$, with probability no smaller than $1-\eta$, the solution $\left(A_{N, k}^{\star}, \boldsymbol{c}_{N, k}^{\star}\right)$ to the scenario optimization problem with constraint removal (7) satisfies

$$
\mathcal{P}\left\{\delta \in \Delta: \boldsymbol{x}_{\delta}(t) \in \mathcal{E}_{\varepsilon}\left(A_{N, k}^{\star}, \boldsymbol{c}_{N, k}^{\star}\right)\right\} \geq 1-\varepsilon
$$

Remark 1. (Choosing an empirical violation parameter $\alpha$ ). The empirical violation probability $\alpha$ is an user-chosen parameter through which the level of approximation of the randomized solution can be tuned. As a matter of fact, though the feasibility of the randomized solution is guaranteed for every $\alpha \in[0, \varepsilon)$, the closer is $\alpha$ to the desired violation probability $\varepsilon$, the better the randomized solution approximates the actual solution to the chanceconstrained problem. At the same time, however, $N$ grows to infinity as $O\left(\frac{1}{\varepsilon-\alpha}\right)$ when $\alpha \rightarrow \varepsilon$, Campi and Garatti [2011], so that one should account for the available computational resources when choosing $\alpha$.

The optimal removal procedure is computationally impracticable, but since Theorem 2 holds irrespectively of the algorithm used to remove the constraints, one can head for suboptimal approaches like removing one by one the constraint that leads to the largest improvement in the cost (greedy removal) or subsequently removing the whole set of active constraints (block removal), i.e., those constraints such that

$$
\left(\boldsymbol{x}_{\delta^{(i)}}(t)-\boldsymbol{c}\right)^{\prime} A\left(\boldsymbol{x}_{\delta^{(i)}}(t)-\boldsymbol{c}\right)=1 .
$$


for the current $(A, \boldsymbol{c})$ solution.

Algorithm 1 implements this latter removal rule for 3D footprint calculation. The shorthand $\boldsymbol{x}_{t}^{(i)}$ is adopted for $\boldsymbol{x}_{\delta^{(i)}}(t)$ for ease of notation.

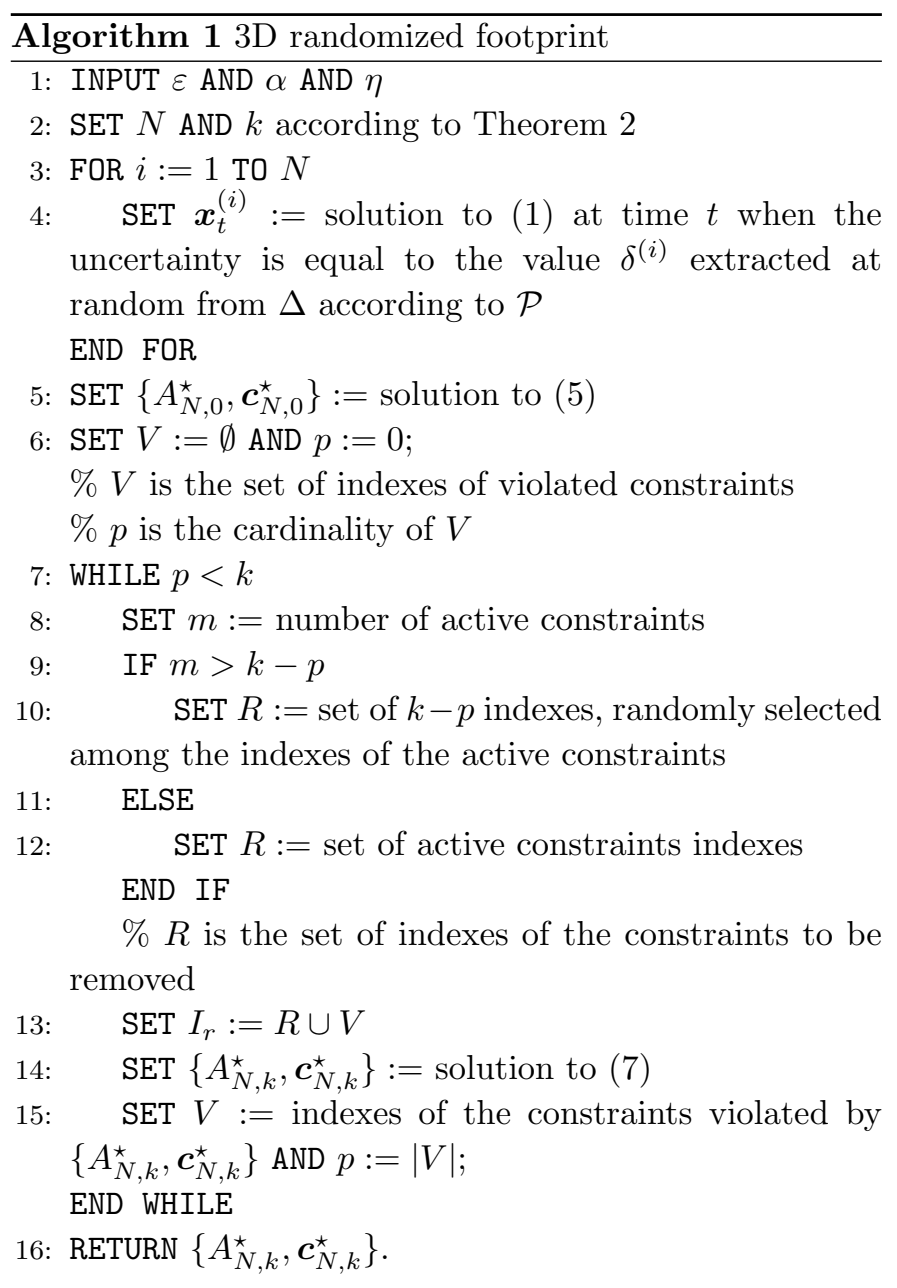

\section{$4 D$ extension}

In order to extend the characterization of the debris dispersion to $4 \mathrm{D}$, we need to account also for time and introduce an ellipsoidal set parameterized by center and shape matrix $(\boldsymbol{c}(t), A(t))$ that are functions of time $t$ in the reference time horizon $\left[t_{i}, t_{f}\right]$. The resulting $4 \mathrm{D}$ chance-constrained problem will be far more challenging to solve than its 3D counterpart, given that the optimization variables $((c)(t), A(t)), t \in\left[t_{i}, t_{f}\right]$, are infinite dimensional.

The solution we opted for is to discretize time and associate to each sample time instant an ellipsoid. The sum of the volumes of all ellipsoids is then minimized subject to the constraint that a fraction of probability at least $1-\varepsilon$ of the debris trajectories belongs to the ellipsoids.

Let $t_{j}, j=1, \ldots, n_{s}$, be the sampled time instants along the reference time horizon $\left[t_{i}, t_{f}\right]$, and $A_{j}$ and $\boldsymbol{c}_{j}$ the parameters of the ellipsoid associated with time $t_{j}$. Then, we have $\min _{\substack{\left(A_{j}, \mathbf{c}_{j}\right), j=1, \ldots, n_{s}}} \sum_{k=1}^{n_{s}} \log \operatorname{det} A_{j}^{-1}$

subject to: $A_{j}=A_{j}^{T} \succ 0, j=1, \ldots, n_{s}$

$\mathcal{P}\left\{\delta \in \Delta: \boldsymbol{x}_{\delta}\left(t_{j}\right) \in \mathcal{E}_{\varepsilon}\left(A_{j}, \boldsymbol{c}_{j}\right), \forall j\right\} \geq 1-\varepsilon$,

a chance-constrained optimization problem which can be solved via scenario approach with constraint removal. The resulting algorithm is similar to Algorithm 1, see Falsone and Noce [2013] for more details.

\section{COMPARATIVE ANALYSIS}

\subsection{Covariance propagation method}

The covariance propagation method proposed in Reyhanoglu and Alvarado [2013] for determining the 4D footprint rests on the linearization of (1) around the nominal trajectory and on the description of the resulting perturbation as a Gauss-Markov process. Dispersion around the nominal trajectory can then be quantified through the ellipsoids representing the level curves of the Gaussian probability density function characterizing the GaussMarkov process.

Set $\boldsymbol{s}=\left[\boldsymbol{x}^{\prime}, \boldsymbol{v}^{\prime}\right]^{\prime}$. Then, (1) can be rewritten in the compact form

$$
\dot{s}=\boldsymbol{f}(\boldsymbol{s})+B \boldsymbol{\xi},
$$

where $B=\left[\begin{array}{lll}0_{3 \times 3} & I_{3 \times 3}\end{array}\right]^{\prime}$. Now, define the perturbation vector $\boldsymbol{z}$ as

$$
z=s-s_{n}
$$

where $s_{n}$ is the nominal trajectory obtained by neglecting $\xi$ and the other sources of uncertainty affecting the system evolution. If we assume that the wind velocity vector $\boldsymbol{w}$ entering (1) through the atmospheric drag deceleration (2) depends on the position only (i.e., $\boldsymbol{w}=\boldsymbol{w}(\boldsymbol{x})$ ), then, the linearized equations governing $\boldsymbol{z}$ are given by

$$
\dot{\boldsymbol{z}}=A(t) \boldsymbol{z}+B \boldsymbol{\xi},
$$

with $A(t)=\left.\frac{\partial \boldsymbol{f}}{\partial \boldsymbol{s}}\right|_{\boldsymbol{s}_{n}(t)}$.

Suppose that the disturbance vector $\boldsymbol{\xi}$ is a white Gaussian noise with mean and covariance given by

$E[\boldsymbol{\xi}(t)]=\overline{\boldsymbol{\xi}}(t), \quad E\left[(\boldsymbol{\xi}(t)-\overline{\boldsymbol{\xi}}(t))(\boldsymbol{\xi}(t)-\overline{\boldsymbol{\xi}}(t))^{\prime}\right]=\Xi(t)$, and it is independent of the initial condition $\boldsymbol{z}()$ at time $t_{i}=0$. If $\boldsymbol{z}(0)$ is Gaussian $\boldsymbol{z}(0) \sim \mathcal{N}\left(\overline{\boldsymbol{z}}_{0}, Z_{0}\right)$, then, (10) describes a continuous time Gauss-Markov process with mean and covariance matrix

$\overline{\boldsymbol{z}}(t)=E[\boldsymbol{z}(t)], \quad Z(t)=E\left[(\boldsymbol{z}(t)-\overline{\boldsymbol{z}}(t))(\boldsymbol{z}(t)-\overline{\boldsymbol{z}}(t))^{\prime}\right]$, that satisfy the following equations:

$$
\begin{aligned}
& \dot{\overline{\boldsymbol{z}}}=A(t) \overline{\boldsymbol{z}}+B \overline{\boldsymbol{\xi}}(t) \\
& \dot{Z}=A(t) Z+Z A(t)+B \Xi(t) B^{\prime}
\end{aligned}
$$

initialized with $\overline{\boldsymbol{z}}(0)=\boldsymbol{z}_{0}$ and $Z(0)=Z_{0}$.

The debris position at time $t$ can then be described as a Gaussian random variable with mean and covariance matrix

$$
\overline{\boldsymbol{x}}(t)=C\left(\overline{\boldsymbol{z}}(t)+s_{n}(t)\right), \quad X(t)=C Z(t) C^{\prime}
$$

where $C=\left[\begin{array}{ll}I_{3 \times 3} & 0_{3 \times 3}\end{array}\right]$.

As a consequence, the 3D ellipsoid containing a fraction $1-\varepsilon$ of the debris trajectories at time $t$ can be determined 
as an appropriate level set of the Gaussian distribution of $\overline{\boldsymbol{x}}(t)$, i.e.,

$$
\mathcal{E}_{\varepsilon}(t):=\left\{x \in \Re^{3}:[\boldsymbol{x}-\overline{\boldsymbol{x}}(t)]^{\prime} X^{-1}(t)[\boldsymbol{x}-\overline{\boldsymbol{x}}(t)] \leq r_{\varepsilon}^{2}\right\},
$$

where $r_{\varepsilon}$ is the Mahalanobis distance between $\boldsymbol{x}$ and $\overline{\boldsymbol{x}}$ and can be computed as the $1-\varepsilon$ quantile of the $\chi^{2}$ distribution with 3 degrees of freedom: $\mathcal{P}\left(V \leq r_{\varepsilon}^{2}\right)=1-\varepsilon$ with $V \sim \chi^{2}(3)$.

The $4 \mathrm{D}$ footprint can then be obtained by varying $t$ within the reference time horizon $\left[t_{i}, t_{f}\right]$ and considering the corresponding ellipsoidal set $\mathcal{E}_{\varepsilon}(t), t \in\left[t_{i}, t_{f}\right]$.

Remark 2. (approximation errors in the CP method). It is worth noticing that there are two sources of approximation in the evaluation of the $4 \mathrm{D}$ footprint according to the outlined procedure: 1) the footprint is constructed based on a linearized model of the system and 2), even if the linearized model were the actual system, there is no guarantee that a fraction of probability $1-\varepsilon$ of the trajectories passes through all the ellipsoidal sets $\mathcal{E}_{\varepsilon}(t), t \in\left[t_{i}, t_{f}\right]$.

\subsection{Numerical example}

In order to compare the Simulation-Based (SB) method proposed in Section 3 with the Covariance Propagation (CP) method, we suppose that the only source of uncertainty is the initial state $s(0)$ at time $t_{i}=0$ and consider the disturbance vector $\boldsymbol{\xi}(t)$ in (1) negligible, as in the simulation results presented in Reyhanoglu and Alvarado [2013].

Results reported in this section refer to the case when the initial state $\boldsymbol{s}(0)$ is given by

$$
\boldsymbol{s}(0)=\boldsymbol{s}_{n}(0)+\boldsymbol{z}(0),
$$

where the nominal initial state is $\boldsymbol{s}_{n}(0)=\left[\boldsymbol{x}_{n}(0)^{\prime}, \boldsymbol{v}_{n}(0)^{\prime}\right]^{\prime}$, with

$$
\begin{aligned}
& \boldsymbol{x}_{n}(0)=\left[0,0,7.8 \times 10^{4}\right]^{\prime} \mathrm{m} \\
& \boldsymbol{v}_{n}(0)=\left[7.0989 \times 10^{3}, 0,-123.9\right]^{\prime} \mathrm{m} / \mathrm{s},
\end{aligned}
$$

and the perturbation to the nominal initial state $\boldsymbol{z}(0)$ is Gaussian with mean $\overline{\boldsymbol{z}}_{0}=0$ and covariance matrix

$$
Z_{0}=\left[\begin{array}{cc}
0_{3 \times 3} & 0_{3 \times 3} \\
0_{3 \times 3} & V_{0}
\end{array}\right],
$$

where $V_{0}=\operatorname{diag}\left(\sigma_{v_{x}}^{2}, \sigma_{v_{y}}^{2}, \sigma_{v_{z}}^{2}\right)$, with $\sigma_{v_{x}}^{2}=\sigma_{v_{y}}^{2}=$ $2500 \mathrm{~m}^{2} / \mathrm{s}^{2}$ and $\sigma_{v_{z}}^{2}=5300 \mathrm{~m}^{2} / \mathrm{s}^{2}$.

The time instants $\left\{t_{j}\right\}_{j=1, \ldots, n_{s}}$ for the $4 \mathrm{D}$ footprint calculation are determined by considering $n_{s}=10$ equally spaced samples of the nominal trajectory $\boldsymbol{s}_{n}(t), t \in\left[t_{i}, t_{f}\right]$, along the $\mathrm{z}$ axis (i.e. the altitude in the ENZ reference frame). Different values for the violation parameter $\varepsilon$ and for the empirical violation $\alpha$ are considered, whereas the confidence parameter $\eta$ is set equal to $\eta=10^{-5}$ in all simulations. The value for $N$ satisfying the bound in Theorem 2 depend on the considered $(\varepsilon, \alpha, \eta)$, the maximal $N$ being $N=10780$.

Table 1 summarizes the results obtained with the SB method and the CP method for a set of values of $\varepsilon$. The value for $\alpha$ used in the SB method is specified in the second column. In all simulations we maintain fixed $N=10780$ and remove $k=\lfloor\alpha N\rfloor$ constraints.

The comparison between the SB method and CP method is in terms of i) volume of the corresponding $4 \mathrm{D}$ footprints (reported in $\mathrm{km}^{3}$ in the last two columns of Table 1) and ii) actual violation $\hat{\varepsilon}$ computed via Monte Carlo simulation by generating a further set of $N$ simulated trajectories and evaluating the fraction of them that exits the $4 \mathrm{D}$ footprint.

\begin{tabular}{c|c||c|c|c|c}
\hline$\varepsilon$ & $\alpha$ & $\hat{\varepsilon}_{N}^{S B}$ & $\hat{\varepsilon}_{N}^{C P}$ & $V^{S B}$ & $V^{C P}$ \\
\hline 0.500 & 0.350 & 0.3505 & 0.5933 & 452.47 & 235.10 \\
0.400 & 0.260 & 0.2602 & 0.5083 & 625.91 & 326.69 \\
0.300 & 0.170 & 0.1705 & 0.4261 & 907.73 & 453.24 \\
0.200 & 0.100 & 0.1003 & 0.3366 & 1304.18 & 646.03 \\
0.100 & 0.035 & 0.0353 & 0.2370 & 2208.56 & 1009.74 \\
0.050 & 0.010 & 0.0103 & 0.1724 & 3552.52 & 1411.29 \\
0.025 & 0.002 & 0.0020 & 0.1328 & 5494.42 & 1846.50 \\
0.020 & 0.001 & 0.0012 & 0.1224 & 6845.50 & 1993.27 \\
0.015 & 0 & $<5 \cdot 10^{-5}$ & 0.1109 & 9182.69 & 2187.03 \\
\hline \multicolumn{5}{c}{ Table 1. Comparative analysis. }
\end{tabular}

Note that if we look at each single row of Table 1, the $4 \mathrm{D}$ footprint volume $V^{S B}$ obtained with the $\mathrm{SB}$ method is larger than volume $V^{C P}$ obtained with the CP method. However, the violation $\hat{\varepsilon}^{C P}$ of the $\mathrm{CP}$ method always exceeds the desired $\varepsilon$ value (possibly due to the approximations errors involved in the method, see Remark 2 ), whereas the violation $\hat{\varepsilon}^{S B}$ of the SB method is always smaller, so that if we compare the volume of the $4 \mathrm{D}$ footprints having the same violation (i.e., $\hat{\varepsilon}^{C P} \simeq \hat{\varepsilon}^{S B}$ ), the SB method outperforms the $\mathrm{CP}$ one. For instance, if we consider the third and sixth rows, $\hat{\varepsilon}^{S B}=0.1705$ and $V^{S B}=907.73$ (third row) and $\hat{\varepsilon}^{C P}=0.1724 \simeq \hat{\varepsilon}^{S B}$ and $V^{C P}=1411.29 \gg V^{S B}$ (sixth row).

As for the SB method, it is worth noticing that actual violation $\hat{\varepsilon}_{N}^{S B}$ is very close to the chosen empirical violation $\alpha$, which affects the size of the $4 \mathrm{D}$ footprint. This is better pointed out in Figure 1, whose plots refer to the same $\varepsilon=0.1$ but different $\alpha$ 's: $\alpha=0$ for the plot on the left and $\alpha=0.035$ for the plot on the right. The debris nominal trajectory is depicted in blue with a solid line, whereas the other simulated trajectories used for the footprint construction are dotted, reporting their samples at the time instants $\left\{t_{j}\right\}_{j=1, \ldots, n_{s}}$. In the plot on the right, some of the simulated trajectories do not belong to the $4 \mathrm{D}$ footprint and are represented through red dots. These are the trajectories that correspond to those constraints that have been removed.

Besides its improved performance with respect to the CP method, the SB method is also applicable to a more general setting, where further sources of uncertainties (like that on the ballistic coefficient or the local wind) are present besides that on the initial velocity.

\section{CONCLUSIONS}

In this work, we studied the problem of estimating the area of the airspace posed at risk by a reentering space debris. We proposed a novel simulation-based method to determine the probabilistic footprint of a single debris fragment, and showed that it outperforms the covariance propagation method recently proposed in the literature. One of the key feature of the introduced simulationbased approach to footprint estimation is that it allows to 

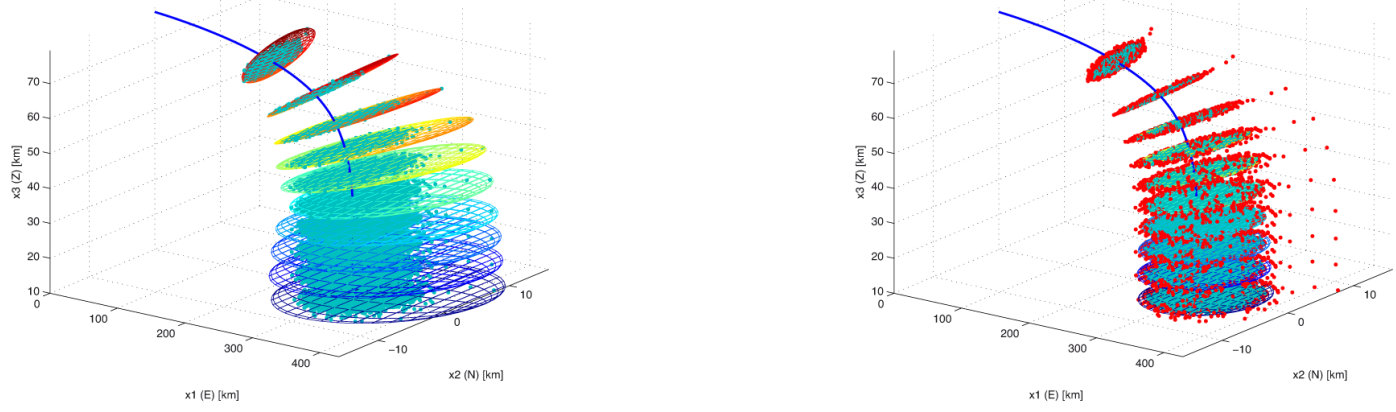

Fig. 1. 4D footprint: simulation-based approach with $\varepsilon=0.1$. Left: $\alpha=0$. Right: $\alpha=0.035$

account for various sources of uncertainty. An interesting direction of research is to consider the breakup event as the starting point for the simulation of a debris cloud. By generating more fragments all together and computing the overall footprint, one can characterize the region of the airspace affected by the debris cloud in toto.

\section{REFERENCES}

Flight safety analysis. Technical Report 1.0, Federal Aviation Administration (FAA), Washington, DC, USA, 2011.

W. Ailor and P. Wilde. Requirements for warning aircraft of reentering debris. Technical report, International Association for the Advancement of Space Safety, 2008.

T. Alamo, R. Tempo, and A. Luque. On the sample complexity of randomized approaches to the analysis and design under uncertainty. pages 4671-4676, Baltimore, MD, USA, June 2010.

L. Anselmo and C. Pardini. Satellite reentry predictions for the Italian civil protection authorities. In 63rd International Astronautical Congress, Naples, Italy, 2013. International Astronautical Federation (IAF).

S. Boyd. Convex Optimization. Cambridge University Press, 2004.

G. Calafiore and M.C. Campi. Uncertain convex programs: randomized solutions and confidence levels. Mathematical Programming, 102(1):25-46, 2005.

G. Calafiore and M.C. Campi. The scenario approach to robust control design. IEEE Transactions on Automatic Control, 51(5):742-753, 2006.

M.C. Campi and S. Garatti. The exact feasibility of randomized solutions of robust convex programs. SIAM Journal on Control and Optimization, 19(3):1211-1230, 2008.

M.C. Campi and S. Garatti. A sampling-and-discarding approach to chance-constrained optimization: feasibility and optimality. Journal of Optimization Theory and Applications, 148(2):257-280, 2011.

M.C. Campi, S. Garatti, and M. Prandini. The scenario approach for systems and control design. Annual Reviews in Control, 33(2):149-157, 2009.
A. Falsone and F. Noce. A randomized approach to the prediction of critical situations for air traffic due to uncontrolled space debris reentry. Master's thesis, Politecnico di Milano - Dipartimento di Elettronica, Informazione e Bioingegneria, 2013.

M.V. Frank, M.A. Weaver, and R.L. Baker. A probabilistic paradigm for spacecraft random reentry disassembly. Reliability Engineering and System Safety, 90(2-3):148161, 2005.

C. Pardini and L. Anselmo. Computational methods for reentry trajectories and risk assessment. Advances in Space Research, 35(7):1343-1352, 2005.

C. Pardini and L. Anselmo. Reentry predictions for uncontrolled satellites: results and challenges. In 6th IAASS Conference - Safety is Not an Option, Montréal, Canada, 2013.

R.P. Patera. Risk to commercial aircraft from reentering space debris. In AAIA Atmospheric Flight Mechanics Conference, 2008.

A. Prèkopa. Stochastic Programming. Kluwer, Boston, MA, 1995.

A. Prèkopa. Probabilistic programming. In A. Ruszczyǹski and A. Shapiro, editors, Stochastic Programming, volume 10 of handbooks in operations research and management science, London, UK, 2003. Elsevier.

M. Reyhanoglu and J. Alvarado. Estimation of debris dispersion due to a space vehicle breakup during reentry. Acta Astronauta, 86:211-218, 2013.

A. Saunders, H.G. Lewis, and G.G. Swinerd. A new tool for satellite reentry predictions. In 5th European Conference on Space Debris, Darmstadt, Germany, 2009. European Space Agency.

P. Sun and R.M. Freund. Computation of minimumvolume covering ellipsoids. Operations Research, 52(5): 690-706, 2004.

M.J. Todd and E.A. Yildirim. On Khachiyan's algorithm for the computation of minimum-volume enclosing ellipsoids. Discrete Applied Mathematics, 155:1731-1744, 2007.

M.A. Weaver, R.L. Baker, and M.V. Frank. Probabilistic estimation of reentry debris area. ESA SP-473, 2:515$520,2011$. 